\title{
Pensamento e Linguagem: Uma Discussão no Campo da Psicologia da Educação Matemática
}

\author{
Mônica Maria Lins Lessa ${ }^{1}$ \\ Universidade Federal Rural de Pernambuco \\ Jorge Tarcísio da Rocha Falcão ${ }^{2}$ \\ Universidade Federal de Pernambuco
}

\begin{abstract}
Resumo
O presente artigo retoma a discussão acerca da relação entre pensamento e linguagem no processo de conceptualização em matemática. Nesse sentido, são oferecidos subsídios empíricos a partir de outros estudos, que permitem discutir e contestar a idéia teórica que propõe um papel central e fundante da linguagem para tal processo de conceptualização. Dados de pesquisa didática referente à compreensão do princípio da equivalência entre equações no ensino introdutório de álgebra permitiram comparar situações de ensino fundadas em competências-em-ação não-verbais versus situações baseadas na explicitação verbal de aspectos relacionados à igualdade entre equações. Os resultados obtidos mostraram ausência de diferenças entre os grupos quanto à apropriação de procedimento algébrico para resolução de problemas, sugerindo complementaridade entre tais métodos. Tais resultados permitem concluir acerca do interesse das representações simbólicas para a conceptualização em matemática, sem com isso excluir ou considerar em plano subalterno aspectos relacionados à ação e à corporeidade na construção do conhecimento matemático.

Palavras-chave: Pensamento e linguagem; competências-em-ação; conhecimento matemático-algébrico.
\end{abstract}

\section{Thought and Language: A Discussion in the Field of Psychology of Mathematics Eucation}

\begin{abstract}
This paper brings back the theoretical debate concerning the relationship between thought and language in the process of mathematical conceptualization. Empirical data issued from other studies are offered for discussing and refusing the idea of assigning a central role to language in concept development and learning in mathematics. These data referred to a didactic research aimed at helping in the understanding of the principle of equivalence between equations, in introductory algebra. Two different didactic contexts were compared: a group working with non-verbal competenciesin-action connected with the principle of equivalence, and another group working on making verbally explicit the meaning of the equality between equations. Results showed no difference in terms of algebraic procedures in problem solving between the two groups. Instead, both methods seem to be complementary. These data confirm the interest of symbolic representation in the process of mathematical conceptualization, neither excluding nor assigning a secondary role to action and corporeality.

Keywords: Thought and language; competences-in-action; algebraic and mathematical knowledge.
\end{abstract}

A discussão teórica sobre as relações entre pensamento e linguagem no âmbito do conhecimento matemático leva necessariamente a uma reflexão epistemológica e psicológica acerca da origem e características básicas do conhecimento (Lakoff \& Núñez, 2000). Em psicologia da educação matemática, o debate teórico tem freqüentemente feito alusão aos seguintes questionamentos: o conhecimento deve ser assimilado fundamentalmente a aspectos lógico-operatórios, e somente de forma secundária a aspectos simbólico-representacionais, ou seria justamente o inverso? Em termos especificamente da conceptualização em matemática, seria pertinente pensar que a aprendizagem da matemática estaria circunscrita à esfera da linguagem, exclusivamente? Alternativamente, não seria pertinente pensar que a construção do conhecimento matemático demandaria a ação do sujeito no mundo físico, de forma não necessariamente mediada pela linguagem (linguagem entendida como recurso à função semiótica, recobrindo desde a utilização de signos lingüísticos orais ou escritos até o apelo a suportes simbólicos de forma geral)? Estes questionamentos são fundamentais quando se

\footnotetext{
${ }^{1}$ Apoio CNPq em forma de bolsa de formação pós-graduada.

Apoio CNPq em forma de bolsa de produtividade em pesquisa.

Endereço para correspondência: Rua Acadêmico Hélio Ramos, s/n, CFCH, $8^{\circ}$ andar, Cidade Universitária, Recife, 56700 901. Fone: (81) 32747330; Fax: (81) 32747331.E-mail:jorge.falcao@pesquisador.cnpq.br/monilins@aol.com
}

trata de refletir acerca da origem psicológica do conhecimento matemático, e tal reflexão, propomos aqui, não pode prescindir das considerações teóricas da perspectiva psicogenética de Jean Piaget, da perspectiva sócio-histórica de Lev S. Vigostki e da perspectiva pós-piagetiana de Gérard Vergnaud (Da Rocha Falcão, 2003b).

Do ponto de vista epistemológico, Piaget defende que o pensamento forma-se a partir dos invariantes operatórios dos esquemas, em um processo de natureza adaptativa, ou seja, biológica (Piaget, 1973b), com clara ênfase sobre aspectos vinculados à ação; em artigo sobre as relações entre linguagem e as operações intelectuais, Piaget afirma existir ... uma lógica de coordenações de ações mais profunda do que a lógica vinculada à linguagem e muito anterior à lógica das 'proposições', no sentido estrito (Piaget, 1973a, p. 63). Nesta mesma ordem de idéias, Piaget admite que a linguagem seria uma condição necessária para a realiraçãa das estruturas lógicas, mas não uma condição sufficiente, pois tratála como suficiente implicaria em desconsiderar o papel fundamental das ações e da inteligência operatória (Piaget, 1973a, p. 63). Nesta perspectiva, o desenvolvimento das operações intelectuais ocorre a partir das ações do sujeito cognoscente, e as raízes das operações lógicas são mais profundas que as raízes das relações lingüísticas. Tal anterioridade da cognição em relação à função simbólica poderia ser exemplificada, na argumentação teórica piagetiana, pelos dados referentes à emergência da permanência de objeto, invariante operatório fundado numa lógica de deslocamentos 
(coordenação de ações) suportada pela imagem mental présimbólica (Piaget, 1977). Coordenações de ações pré-simbólicas estariam igualmente na base do estabelecimento de relações de ordem e de relações parte-todo, fundamentais para a construção, respectivamente, dos invariantes da seriação e transitividade, e dos invariantes relacionados à inclusão de classes (Piaget, 1973a). Conforme os exemplos acima sugerem, os invariantes lógicooperatórios se estruturariam a partir das ações sensório-motoras, constituindo o pensamento (Piaget, 1973a). Portanto, conforme ressalta Banks-Leite (1997), enquanto estruturada pela lógica, a linguagem permanece subordinada à formação das operações lógico-matemáticas, embora contribua necessariamente para o 'fechamento' das estruturas formais (pp. 212-213). Assim, a perspectiva piagetiana propõe, em última análise, que o desenvolvimento do pensamento se inicia no período sensório-motor, caracterizado como um período prélingüístico, de tal sorte que a linguagem passa a ser uma subesfera do pensamento, e não o contrário.

$\mathrm{Na}$ perspectiva denominada sócio-culturalista ou sóciohistórica, Vigotski (2001) defende que o pensamento forma-se a partir de ferramentas mediacionais simbólicas. Para este autor, o processo de desenvolvimento cognitivo não segue padrões estruturais gerais de natureza biológico-adaptativa, ... como sombra lançada pelo objeto, sem coincidir com ele mas reproduzindo com precisão e repetindo seu movimento (p. 245). Nesse sentido, o aporte simbólico proporcionado à criança por seu contexto sócio-cultural imediato (aí incluída a escola) e o desenvolvimento geral não representam dois processos independentes, mas um único processo no contexto do qual estes dois aspectos se inter-relacionam de forma complexa. Nessa linha de formulação, o pensamento não pode ser concebido enquanto atividade exclusivamente autônoma, individual, auto-referente. Em conseqüência, a psicologia cognitiva não poderia limitar-se à abstração do intrapsíquico, uma vez que, conforme assinala Bronckart (1985), ... o pensamento e a consciência não constituem uma emanação de características estruturais ou funcionais internas ..., mas são, ao contrário, determinadas pelas atividades externas e objetivas realizadas com os congêneres, num ambiente social determinado (p. 14).

Para autores considerados pós-piagetianos, como Gérard Vergnaud, propositor da teoria dos campos conceituais (Vergnaud, 1990), a função simbólica não seria nem uma condição necessária nem uma condição suficiente do processo de conceptualização em geral (e conceptualização matemática em particular), mas contribuiria decisivamente para esse mesmo processo, notadamente em termos da transformação tanto de competências práticas (conhecimentos-em-ação), quanto de categorias genéricas de pensamento em objetos matemáticos ${ }^{4}$. Este referencial teórico, ao explorar as relações entre pensamento e linguagem, tem oferecido subsídios importantes para as reflexões acerca da passagem do pensamento aritmético para o pensamento algébrico, conforme discutido por Da Rocha Falcão (1997). Apesar de claramente alicerçada em pressupostos teóricos

\footnotetext{
${ }^{4}$ Ver a esse respeito reflexão proposta Nunes $(1994,1997)$.
}

piagetianos, a perspectiva vergnausiana avança em relação àquela em função basicamente de três aspectos: em primeiro lugar, defende uma perspectiva acerca da constituição do conhecimento (ou mais especificamente da conceptualização) que vai além do primado dos invariantes lógico-operatórios (I) para a formação dos conceitos, acrescentando a tais aspectos outros dois pontos constitutivos do chamado tripé teórico da conceptualização, quais sejam os suportes de representação $(\mathrm{R})$ e as situações sócio-culturalmente significativas (S), no contexto das quais o conhecimento é mobilizado e utilizado. Em segundo lugar, tal perspectiva estabelece claramente a necessidade de se levar em conta a especificidade dos conteúdos de conhecimento, ou seja, a referência dos mesmos a determinado campo epistêmico circunscrito (Ex.: matemático, físico, moral, histórico, etc). Tal proposição, conforme discutir-se-á mais adiante, terá conseqüências importantes em termos das questões relacionadas à aprendizagem e seus obstáculos, pois estes não mais serão restritos a estruturas gerais de pensamento, mas terão de levar em conta aspectos dificultadores inerentes àquele domínio específico. Um exemplo dentre vários, nesse sentido, seria a construção de significado para o sinal de igualdade em álgebra, conforme descrito por Analúcia Schliemann e cols. (Schliemann, 1992). Em terceiro e último lugar, tal perspectiva enfatiza a importância do conceito teórico de esquema, definido como organização invariante da conduta para uma classe delimitada de situações (Vergnaud, 1990). O esquema estaria aqui na base tanto de competências conceituais, necessariamente baseadas em suportes simbólicos (além de invariantes e situações), como de competências práticas não explicitáveis simbolicamente (conhecimentos-em-ação e/ou teoremas-em-ação), como, p. ex., a competência de crianças brincarem em gangorras compensando eventuais diferenças de peso entre cada uma das duas crianças na gangorra, com a mais pesada sentando-se consistente e deliberadamente mais próxima do ponto de apoio da gangorra, sem, contudo dispor de nenhuma explicação $0^{5}$ para tal iniciativa. Para Vergnaud (1990), a linguagem teria importância decisiva na passagem dos esquemas tipo competência-em-ação para os esquemas conceituais, pois tal passagem decorreria necessariamente da explicitação das referidas competências, ou seja, da aquisição de competência discursiva integrada à competência prático-funcional.

A partir da proposição do esquema como elemento teórico unificador de competências cognitivas conceituais e préconceituais, Da Rocha Falcão (1997) propõe análise da construção de significado em contexto matemático específico, a álgebra. Para este autor, tal campo conceitual fornece excelente exemplo de concatenação entre representação e invariantes operatório (cf. dados discutidos em Brito Lima \& Da Rocha Falcão, 1997), bem como entre competências-em-ação (como a habilidade de pesar produtos utilizando-se balanças de dois pratos, em contexto extra-escolar de comércio) e competências simbólico-conceituais, como o princípio da equivalência em

${ }^{5}$ Tal explicação poderia basear-se no Princípio Universal das Alavancas, proposto inicialmente por Arquimedes. Tal princípio é freqüentemente discutido na escola fundamental, porém raramente conectado de fato, na mente dos alunos, à brincadeira da gangorra ou a qualquer outra brincadeira ou atividade extra-escolar. 
álgebra (dada uma determinada equação algébrica, pode-se obter equação dela derivada e equivalente desde que se opere de forma semelhante sobre os dois membros da mesma) (cf. Da Rocha Falcão, 1995; Lins Lessa, 1996). As representações simbólicas, segundo Da Rocha Falcão (1995, 1997), constituem-se em aspecto-chave na aprendizagem da matemática devido notadamente a dois aspectos: em primeiro lugar, elas não se restringem a uma mera roupagem de estruturas operatórias previamente dadas, e em segundo, tais representações servem de elemento vinculador do indivíduo a determinado acervo cultural (cf. ilustrado pela balança de dois pratos), dando ao processo de construção conceitual um componente necessariamente histórico-cultural (Da Rocha Falcão, 2003a) Tais concepções estão alicerçadas na teoria dos campos conceituais, proposta por Vergnaud (1990), a qual defende que o suporte representacional não somente reveste, mas participa diretamente da geração do conceito, e que tal conceito é necessariamente simbólico, pois se constrói e é explicitado através da linguagem.

Tal perspectiva teórica tem, portanto, clara herança piagetiana, a qual se enriquece de aportes oriundos da perspectiva sócioculturalista vigotskiana. Sabemos que as perspectivas piagetiana e vigotskiana assumem pressupostos e se enraízam em tradições filosófico-epistêmicas claramente distintas (cf. Bruner, 1997), mas ao mesmo tempo não nos parece desejável, conforme discute Nunes (2000), que o fenômeno humano, enquanto criatura teórica piagetiana, não possa se beneficiar dos aportes de outra construção, a criatura teórica vigotskiana. Tal esforço parece-nos desejável em psicologia da educação matemática, conforme tentaremos mostrar através de estudo empírico realizado por Lins Lessa (1996) acerca da construção do conhecimento matemático, também no campo conceitual da álgebra. Espera-se, com tais dados, contribuir para a discussão acerca da conceptualização em álgebra, e em última análise, para a discussão acerca possibilidades e limites da linguagem na construção do conhecimento matemático.

\section{Estudo empírico}

Em estudo anterior, Lins Lessa (1996) se propôs a analisar situações didáticas potencialmente facilitadoras para a introdução ao campo conceitual da álgebra, procurando investigar que aspectos desses conceitos seriam mais bem contemplados por determinados suportes didáticos. Nesse sentido, elegeu-se um aspecto fundamental na construção de significado em álgebra, qual seja a compreensão do princípio da equivalência (Vergnaud, Cortez \& Favre-Artigue, 1987), segundo o qual pode-se passar de uma equação para outra dita equivalente se tal passagem decorrer da realização de uma mesma operação em ambos os membros da referida equação. No que diz respeito aos suportes didáticos facilitadores de tal compreensão, verificou-se comparativamente o interesse da mobilização da balança de dois pratos, bem como a proposição de problemas verbais enfatizando em sua apresentação a igualdade entre os membros da equação. Para a realização da análise comparativa em questão, verificou-se sistematicamente o efeito do treinamento em resolução de problemas envolvendo igualdades em duas condições (Ex.: Figuras 1 e 2): a) condição de igualdade entre objetos em equilíbrio em uma balança de dois pratos; e, b) condição de igualdade entre quantidades enfatizadas no enunciado de problemas verbais.

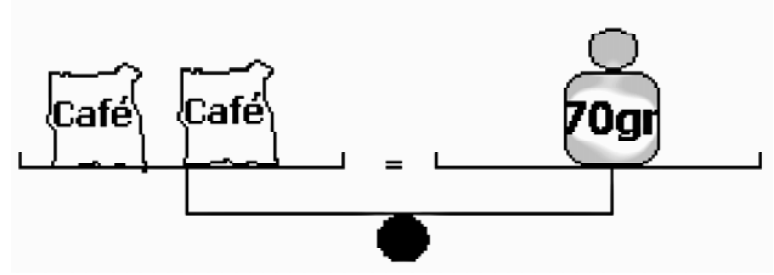

Figura 1. Grupo A, exemplo de uma situação-problema na condição de treinamento balança de dois pratos.

Amanda e Tiane colecionavam papéis de carta. Amanda tinha 70 papéis de carta. Tiane tinha 10 papéis de carta e ganhou dois blocos do seu pai. As duas meninas ficaram com a mesma quantidade de papéis de carta na coleção. Sabendo-se que os blocos tinham a mesma quantidade de papéis, quantos papéis de carta tinham em cada bloco?

Figura 2. Grupo B, exemplo de um problema verbal enfatizando a igualdade na condição de treinamento problemas verbais (itáliconegrito acrescentado no exemplo acima para fins de indicação).

O estudo foi constituído de três fases, cada uma das quais realizada em uma sessão de entrevista, em que o examinador entrevistou individualmente 40 participantes (sendo 20 para o Grupo A - Balança, e 20 para o Grupo B - Problemas Verbais) na faixa etária de 11 e 12 anos, cursando a $5^{\text {a }}$ série do ensino fundamental de escolas da cidade de Recife (PE). A primeira fase consistiu em um pré-teste, onde os participantes resolveram uma lista de seis equações e seis problemas verbais envolvendo igualdades entre quantidades. A segunda fase consistiu em um treinamento, em que os participantes foram submetidos individualmente a uma série de 12 tarefasproblema. Nesta fase cada um dos grupos (A e B) participou de uma das duas condições de treinamento acima referidas (Grupo A: Balança e Grupo B: Problemas Verbais). Na terceira fase, foi realizado o pós-teste, em que os participantes resolveram a mesma lista de equações e problemas verbais resolvidos no pré-teste. Em todas as fases os participantes deveriam descobrir o valor das incógnitas envolvidas e justificar a sua resposta tentando explicitar o raciocínio utilizado. Foram estudadas seis estruturas algébricas nos problemas, duas mais simples (estruturas 1 e 2), que poderiam ser resolvidas pela aritmética, e quatro mais complexas, para as quais a utilização da álgebra se faz necessária (Tabela 1). 
Tabela 1

Estruturas Matemáticas Subjacentes aos Problemas Utilizados no Treinamento

\begin{tabular}{cc}
\hline Estrutura & Representação Simbólica \\
\hline 1 & $\mathrm{ax}+\mathrm{b}=\mathrm{c} ; \mathrm{ax}=\mathrm{b}$ \\
2 & $\mathrm{ax}+\mathrm{x}=\mathrm{b}$ \\
3 & $\mathrm{a}+\mathrm{x}=\mathrm{bx}$ \\
4 & $\mathrm{ax}+\mathrm{b}=\mathrm{cx}+\mathrm{d}$ \\
5 & $\mathrm{ax}+\mathrm{b}=\mathrm{ax}+\mathrm{cy}+\mathrm{d}$ \\
6 & $\mathrm{ax}+\mathrm{by}+\mathrm{c}=\mathrm{dx}+\mathrm{by}+\mathrm{f}$ \\
\hline
\end{tabular}

Para compreender melhor os resultados desse estudo, fazse necessário um comentário sobre a categorização dos dados e apresentação de algumas figuras. As produções dos participantes foram categorizadas, tanto no pré como no pósteste, a partir de três aspectos básicos: tipo de resposta, referente à análise clássica de desempenho, utilizando-se apenas as categorias acerto-não-acerto; tipo de procedimento, referente à forma básica (aritmética ou algébrica) de resolução mobilizada para resolver o problema; e modos de representação, referentes aos suportes representacionais auxiliares mobilizados pelos participantes. No presente artigo escolheu-se analisar exclusivamente os itens de categorização tipo de procedimento, que será analisado a seguir, e o modo de representação, que será analisado após a análise do tipo de procedimento. Tais itens são discutidos a baixo.

\section{Tipo de Procedimento}

Conforme explicitado, consistiu na análise da estratégia usada na resolução dos problemas e equações, tendo-se classificado as produções dos participantes em termos de três categorias: procedimento aritmético, procedimento intermediário e procedimento algébrico. Vale salientar aqui que o objetivo central do treinamento proposto foi discutir o procedimento algébrico para que o participante passasse a optar pela utilização desse procedimento para resolver problemas e equações. Em termos da categorização de procedimento já referida, proceder algebricamente consistiu em realizar uma manipulação algébrica sobre a expressão matemática proposta, isto é, realizar operações semelhantes em ambos os membros da igualdade, a partir de uma representação explícita ou de uma representação mental. $\mathrm{O}$ extrato de protocolo 1 dá um exemplo do que foi considerado como procedimento algébrico:

Participante $\mathrm{n}^{\circ} 10$

(extrato de protocolo: problema 3/ procedimento algébrico / pósteste):

E: Me explica como você fez!

P: Fábio tinha 69 bolas mais um pacote da sua mãe, e Fernando tinha ganhado quatro pacotes de bolas de gude, se eles tinham a mesma quantidade, cortei o pacote de bolas que a mãe deu para Fábio, um eu cortei, e de Fernando eu cortei, então quer dizer que esses três que sobraram é igual a essa quantidade aqui (69).

$\cdots$
E: agora, por que tu cortaste um de um lado e outro do outro lado?

P: porque os dois são da mesma quantidade

E: e cortando continua igual?

P: continua igual

E: por quê?

P: Porque, tirando a mesma quantidade, da mesma forma, continua igual.

O procedimento aritmético, por sua vez, consistiu na abordagem direta dos problemas, sem manipulações intermediárias e buscando imediatamente o estabelecimento do valor da incógnita, seja por atribuições aproximadas de valores, seja através da realização de operações preliminares, até a obtenção do valor procurado. O extrato de protocolo abaixo ilustra tal tipo de procedimento:

Participante $\mathrm{n}^{\mathrm{o}} 35$

(extrato de protocolo: problema 1/procedimento aritmético / pósteste):

E: Leonardo e Rafael colecionavam o álbum ... (leitura do problema)

quantas figurinhas tinham em cada pacote? Vai fazer o quê?

P: 47 menos 23 dividido por 2.

E: Por que você vai pegar o 47 ?

P: Porque eu pegava 47 subtrai e o resultado eu dividia por 2.

E: E Leornardo tem quantas figurinhas ao todo?

P: 23.

E: Ele só tem as 23?

P: Não, 47.

E: Ao todo?

P: É, ele tem ao todo 47 figurinhas.

E: Igual ou diferente de Rafael?

P: Igual

E: Então tem que descobrir quantas figurinhas tem no pacote!

P: (Escreveu no protocolo)

$\mathrm{E}:$ Tem quantas figurinhas em cada pacote?

P: 12.

E: Ao todo Leonardo tinha quantas figurinhas?

P: 47.

E: E Rafael?

P: 47.

E: Os dois meninos tinham a mesma quantidade de figurinhas na coleção?

P: Tinham.

A análise dos resultados obtidos a partir da categorização permitiu verificar que o procedimento aritmético predominou no pré-teste, enquanto que o procedimento algébrico predominou no pós-teste, tanto no grupo A (Grupo de Treinamento com a Balança), quanto no grupo B (Grupo de Treinamento com os Problemas Verbais). Tais resultados demonstram que o treinamento cumpriu seu objetivo de tornar o procedimento algébrico elegível para a resolução de determinada classe de problemas (aqueles com estruturas matemáticas de tipo algébrico). Todavia, é importante destacar que o procedimento 
aritmético apresenta ainda um percentual considerável no pósteste, o que se deve às estruturas 1 e 2 , as quais continuam sendo resolvidas por esse procedimento (Figuras 3 e 4).

A partir da análise das Figuras 3 e 4, constata-se que o procedimento algébrico é predominantemente utilizado pelos participantes nos dois grupos de treinamento, sugerindo que ambos os tipos de suporte didático (balança ou problemas verbais) promovem a passagem do procedimento aritmético para o procedimento algébrico, isso para aqueles problemas cuja estrutura matemática subjacente seja efetivamente algébrica. Ou seja, o tipo de procedimento depende do tipo da estrutura envolvida na equação ou no problema.

Então, o que parece ser mais importante para iniciação à álgebra, ou seja, a instrumentalização conceitual dos alunos de forma a habilitá-los a adotar um procedimento algébrico na resolução de problemas/equações, não é o suporte didático em si, mas tal suporte combinado a problemas de estrutura algébrica. Nesse sentido, são as estruturas 3, 4, 5 e 6 (incógnitas aparecendo nos dois membros da equação) que favorecem a compreensão de conceitos básicos na introdução à álgebra (igualdade, representação, incógnita e manipulação de incógnitas). Quando se trata das estruturas mais simples (Ex.: estruturas 1 e 2), a estratégia usada é aritmética e quando se trata das estruturas mais complexas (Ex.: estruturas 3, 4, 5 e 6), usa-se o procedimento algébrico. Como afirma Vergnaud (1988), não há necessidade de introduzir a álgebra para resolver problemas que envolvem as estruturas simples (incógnita em apenas um membro da equação), pois nestes casos procedimento algébrico não oferece nenhum benefício. Isto pode ser confirmado nas figuras 5 e 6 a seguir:

Um outro aspecto apontado por este estudo é que o modo de representar os problemas depende do tipo de treinamento recebido. Além disso, é possível estabelecer uma relação entre o procedimento algébrico e o modo de representação utilizado. Tais conclusões são discutidas a seguir.

\section{Modo de Representação}

Tal item de categorização consistiu na análise da representação auxiliar proposta pelos participantes na resolução dos problemas, conforme resumido na Tabela 2. Observa-se que no grupo A aparecem diferentes formas de representação (desenho, verbal e literal), mas o conjunto delas apresenta um percentual menor (43,74\%) do que o uso das contas (49,17\%). No grupo B o conjunto dessas representações apresenta um percentual maior $(64,58 \%)$ do que as contas (32,92\%) (Figuras 7 e 8).

Considerando a relação entre o modo de representação e 0 procedimento algébrico para resolução dos problemas e equações, parece que o uso do procedimento algébrico pressupõe uma forma de representação, sendo esta mais simbólica (Verbal 16,67\%; Literal 25,41\%) quando o treinamento ocorre com os problemas verbais (Grupo B). Quando o treinamento ocorre com a balança os percentuais da representação simbólica caem (Verbal 8,75\%; Literal 7,91\%; Exam.faz $7,09 \%$ e o que aparece com maior frequência é a representação

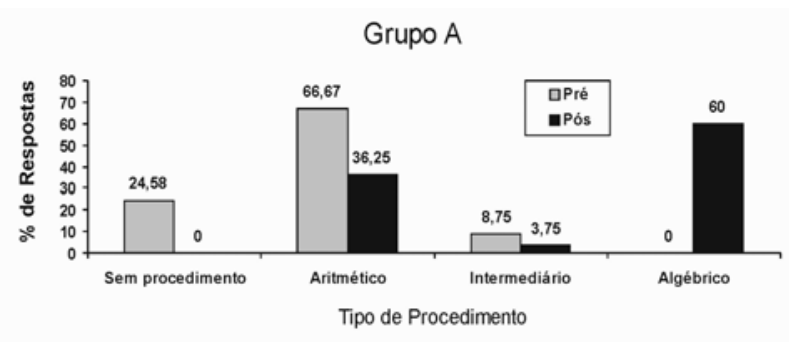

Figura 3. Distribuição da freqüência percentual das categorias de resposta no item tipo de procedimento (Grupo A: Balança).

\section{Grupo B}

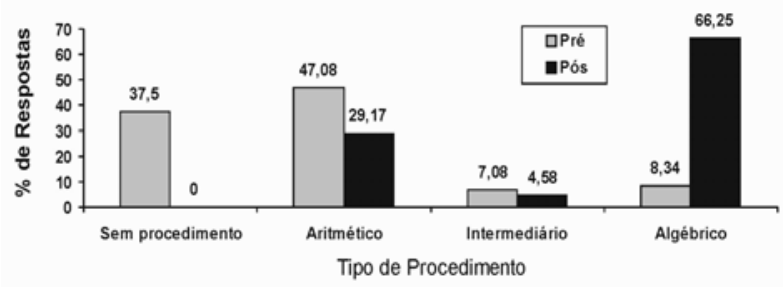

Figura 4. Distribuição da freqüência percentual das categorias de respostas no item tipo de procedimento (Grupo B: Problemas Verbais).

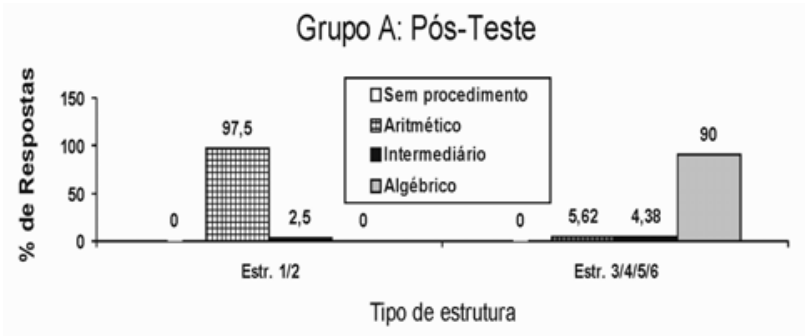

Figura 5. Distribuição da freqüência percentual do tipo de procedimento nos diferentes tipos de estrutura (Grupo A - Balança: Pós-teste). Legenda: Estr.1/2 = estrutura 1 e estrutura 2; Estr. 3/4/ $5 / 6=$ estrutura 3 , estrutura 4 , estrutura 5 e estrutura 6.

\section{Grupo B: Pós-Teste}

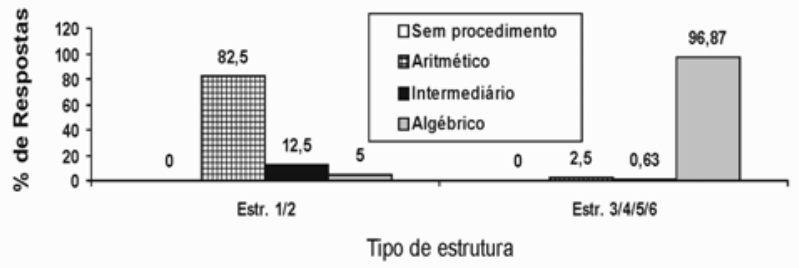

Figura 6. Distribuição da freqüência percentual do tipo de procedimento nos diferentes tipos de estrutura (Grupo B - Problemas Verbais: Pós-teste). Legenda: Estr.1/2 = estrutura 1 e estrutura 2; Estr. $3 / 4 / 5 / 6=$ estrutura 3 , estrutura 4, estrutura 5 e estrutura 6. 
Tabela 2

Modos de Representação Propostos pelos Participantes como Auxiliares a seus Procedimentos de Resolução dos Problemas Utilizados no Treinamento

\begin{tabular}{ll}
\hline Modo de representação & Descrição \\
\hline Sem representação explícita & Participantes da categoria "não fez, deixou em branco", quanto ao procedimento. \\
Desenho & Desenho da balança + pacotes (Grupo A) ou caixas/ pacotes (Grupo B) \\
Verbal & Resumo do problema, abreviado ou não (linguagem natural escrita) \\
Literal & Uso de letras como x, y, z \\
Contas & Operações aritméticas sem um plano aparente
\end{tabular}

(Sem representação auxiliar explicitada)

O examinador propõe a representação Desenho da balança + pacotes (Grupo A) ou desenho de caixas/pacotes (Grupo B)

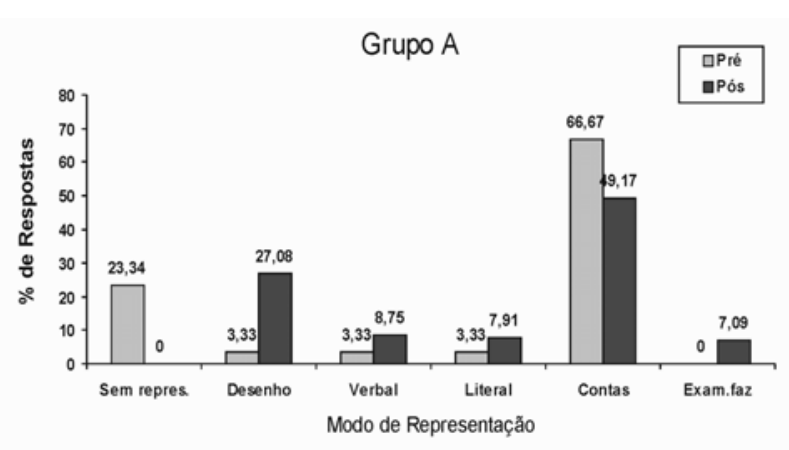

Figura 7. Distribuição da freqüência percentual do modo de representação - Grupo A (Balança). Legenda: Sem repres. = não resolve/protocolo em branco; Contas = apenas operações aritméticas; Exam. faz = examinador faz a representação

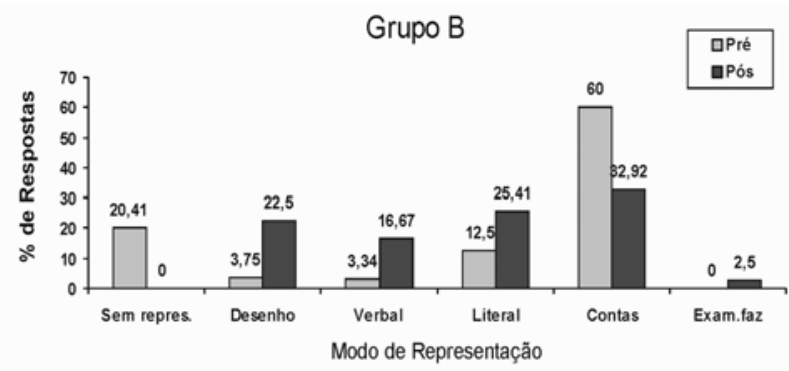

Figura 8. Distribuição da freqüência percentual do modo de representação - Grupo B (Problemas Verbais). Legenda: Sem repres. $=$ não resolve/protocolo em branco; Contas $=$ apenas operações aritméticas; Exam. faz = examinador faz a representação.

em forma de desenho $(27,08 \%)$ no Grupo A, destacando que a representação icônica é a mais próxima do objeto real (a balança). Além disso, o percentual em 'contas' é maior neste grupo. Estes resultados parecem indicar que suporte representacional desempenha um papel importante para o raciocínio e procedimento algébrico, e que cada tipo de treinamento oportuniza a compreensão de determinados aspectos do campo conceitual algébrico: a) a balança oportuniza a compreensão do princípio de equivalência, de incógnita, $a$ experimentação da manipulação de incógnitas no campo da ação; e, b) os problemas verbais oportunizam a compreensão de igualdade, incógnita e manipulação de incógnita no campo da representação simbólicoformal.

\section{Discussão e Conclusão}

O presente artigo teve como objetivo central retomar a discussão acerca da relação entre pensamento e linguagem, contextualizando tal discussão no âmbito da construção do conhecimento matemático, mais especificamente, a aprendizagem da álgebra. Neste sentido, os dados do estudo empírico proposto por Lins Lessa (1996) foram analisados, de forma a subsidiar tal discussão. Dentre os aspectos abordados a partir dos resultados obtidos em tal estudo, dois parecem merecer destaque: 1) a natureza da construção do princípio da equivalência e a compreensão da manipulação de incógnitas a partir da ação do participante na condição de treinamento com a balança de dois pratos; e, 2) a natureza proposicional envolvida na representação dos problemas.

Discutindo o primeiro aspecto é possível comentar que durante as sessões de treinamento com a balança os participantes tinham a oportunidade de observá-la concretamente, ou seja, eles podiam observar quando ela estava em equilibrio e em desequilibrio. Além disso, os participantes tinham a oportunidade de manipular a balança a fim de obter o equilíbrio ou o desequilibrio através da sua ação sobre a mesma. Nesta situação de treinamento o discurso do examinador, por exemplo, se eu coloco o mesmo peso em ambos os pratos da balanç, ela fica equilibrada, se a balança estiver equilibrada e eu tirar o mesmo peso de cada lado, ela vai continuar equilibrada, tinha como suporte o objeto onde os participantes podiam constatar empiricamente o que estava sendo verbalizado. Sendo assim, pode-se dizer que a construção do princípio da equivalência, de incógnita e de manipulação de incógnita teve como suporte auxiliar a ação do participante sobre o objetobalança. Isso não quer dizer que a construção de conceitos decorra da ação concreta do participantes e não da fala do examinador, e sim, que a situação concreta deu suporte à fala do examinador, uma vez que a balança, enquanto artefato da cultura, mobiliza conhecimentos práticos extra-escolares referentes à noção do equilibrio, com sua aplicação em situações culturais de pesagem e comércio. Poder-se-ia dizer que a construção de significados algébricos ocorrida no contexto da atividade com a balança teria como suporte preponderante (ou conceptual scaffolding - andaime conceitual, segundo Da Rocha Falcão, 1995) uma ação (no sentido piagetiano do termo) e não uma representação mediacional.

Diferentemente do treinamento com a balança, no treinamento com os problemas verbais, o examinador e o participante estavam diante de uma atividade que envolvia um suporte representacional. 
Quando o examinador fazia referência à igualdade entre quantidades enfatizadas no enunciado de problemas verbais, o seu discurso tinha como suporte uma proposição verbal que envolvia uma estrutura lógica, por exemplo: As Lojas Americanas e Lobrás tinham a mesma quantidade de chocolate, as caixas de chocolate da Lobrás eram iguais às caixas das Lojas Americanas, vamos supor que as Lojas Americanas tivessem vendido uma caixa e a Lobrás também tivesse vendido, as lojas iam continuar com a mesma quantidade?".

Tal suporte poderia igualmente vir na forma de uma equação escrita no papel, como ilustrado nas equações reproduzidas no quadro 2. Nestes casos, a construção do princípio de equivalência não estava apoiada em metáforas conceituais corporificadas em artefatos da cultura, e sim numa estrutura lógica proposicional do tipo se ... então, que demanda esquemas operatórios formais do pensamento. Nesta situação, poder-se-ia dizer que a mediação simbólica ocorrida na atividade com os problemas verbais estava alicerçada num suporte representacional discursivo. Estes dados vão na mesma direção dos dados do estudo de Brito Lima e Da Rocha Falcão (1997), em que a proposição representacional prévia do problema algébrico através de um desenho facilitou grandemente a abordagem e resolução do mesmo.

Ao considerarmos comparativamente a situação da balança e a situação dos problemas verbais com ênfase na igualdade, verificamos que tanto a ação quanto o discurso oferecem suportes auxiliares na resolução de problemas algébricos. Portanto, o que está em questão aqui não é a defesa do material concreto como suporte auxiliar, no sentido de que a criança precisa desses materiais para construir conceitos lógico-matemáticos. Até porque os resultados do estudo apontaram que não houve diferença entre os dois grupos quanto ao uso do procedimento algébrico - trabalhado durante as duas condições de treinamento - para resolver os problemas e as equações. A questão é que o tipo de suporte didático (concreto-metafórico versus simbólico-discursivo) não é o aspecto mais importante na iniciação ao ensino da álgebra. $\mathrm{O}$ que o estudo evidencia é que com a balança a criança pode compreender o princípio de equivalencia a partir de esquemas sensóriomotores, mas com os problemas ela precisa de esquemas operatórios formais para compreender o mesmo princípio. Isto é, numa situação (balança) o discurso não é fundamental (ou suficiente) para compreensão do princípio, o discurso organiza e dá significado à ação, mas na outra situação (problemas verbais) a compreensão do princípio se dá a partir dele. Em suma, como afirma Piaget, a linguagem permanece como condição necessária do acabamento das estruturas lógicas, pelo menos no nível das estruturas proposicionais, mas nem por isso constitui uma condição suficiente de sua formação (Piaget, 1973a, p. 63).

Portanto, os dados do estudo empírico apontam para a possível construção do conhecimento matemático sem ser necessariamente mediado em primeira instância pela linguagem. Tais dados nos autorizam, portanto a propor que, se o conhecimento matemático abarca desde competências-em-ação, teoremas-em-ação e esquemas, que não são necessariamente conceituais, então a construção desse conhecimento não necessariamente é mediada pela linguagem. Em se tratando da álgebra que consiste num conjunto de princípios, conceitos, regras e procedimentos matemáticos para a modelização e resolução de problemas especificos, cuja abordagem por outros métodos (Ex.: aritméticos) seria difícil ou impossivel (Da Rocha Falcão, 1993), constata-se a importância da linguagem na construção desse conhecimento. Porém, o fundante dos esquemas conceituais abstratos é a ação (esquema sensório-motor) e, portanto, a noção de esquema_pressupõe a interação entre os aspectos simbólicos e operatórios num contexto específico, como por exemplo, a construção do conhecimento algébrico.

Finalmente, o ponto de vista teórico que se pretende defender acerca da construção do conhecimento matemático é que o mesmo não pode ser considerado exclusivamente em termos simbólico-proposicionais ${ }^{6}$. Determinados aspectos conceituais (como o princípio da equivalência em álgebra) não podem ser restritos à lógica proposicional, pois o aspecto fundante de tal construção de significado remonta a esquemas sensório-motores, por sua vez enraizados na ação e corporeidade (Lakoff \& Núnez, 2000) do participante.

\section{Referências}

Banks-Leite, L. (1997). As questões lingüísticas na obra de Jean Piaget: Apontamentos para uma reflexão crítica. Em L. Banks-Leite (Org.), Percursos piagetianos (pp. 207-224). São Paulo: Cortez.

Brito Lima, A. P. \& Da Rocha Falcão, J. T. (1997). Early development of algebraic representation among 6-13 year-old children: The Importance of didactic contract. XXI International proceedings of the XXVI International Conference for the Psychology of Mathematics Education (Vol. 2.; pp. 201-208). Lahti, Filândia. Bronckart, J. P. (1985). Vygotski aujourd'bui. Neuchâtel: Delachaux \& Niestlé.

Bruner, J. (1997) Celebrating divergence: Piaget and Vygotsky. Human Development, 40, 63-73.

Da Rocha Falcão,J. T. (1993). A álgebra como ferramenta de representação e resolução de problemas. Em A. D. Schliemann, D. W. Carraher, A. G. Spinillo, L. L. Meira e J. T. Da Rocha Falcão (Orgs.), Estudos em psicologia de educação matemática. Recife: Editora Universitária UFPE.

Da Rocha Falcão, J. T. (1995). A case study of algebraic scaffolding: From balance scale to algebraic notation. Proceedings $O f$ The XXI International Conference for the Psychology of Mathematics Education (Vol. 2; pp. 66-73). Recife, PE.

Da Rocha Falcão, J. T. (1997). Lenguaje algebraico: Un enfoque psicológico. UnoRevista de Didáctica de las Matemáticas, 14, 25-38.

Da Rocha Falcão, J. T. (2003a). Relações entre Pensamento e Linguagem: Explorações teóricas no contexto da educação matemática. Boletim Gepem, 41, 43-56.

Da Rocha Falcão, J. T. (2003b). Contribuições do paradigma construtivista para a psicologia da aprendizagem da matemática. Vetor Neteclem, 1, 69-94.

Frade, C. (2003). Componentes tácitos e explícitos do conhecimento matemático de áreas e medidas. Tese de Doutorado não-publicada, Programa de Pós-Graduação em Educação, Universidade Federal de Minas Gerais. Belo Horizonte, MG.

Lakoff, G. \& Núñez, R. E. (2000). Where mathematics comes from: How the embodied mind brings mathematics into being. New York: Basic Books.

Lins Lessa, M. M. (1996). Balança de dois pratos e problemas verbais como ambientes didáticos para inciação à allgebra: Um estudo comparativo. Dissertação de Mestrado não-publicada, Curso de Pós-Graduação em Psicologia Cognitiva, Universidade Federal de Pernambuco. Recife, PE.

${ }^{6}$ Tal convicção teórica é compartilhada por outros pesquisadores em educação matemática, conforme discutido e empiricamente fundamentado por Frade (2003). Do ponto de vista teórico, ver igualmente a discussão acerca da diferenciação entre conhecimentos tácitos (implícitos, informais) e explícitos (formais, conceituais), bem como a importância dos primeiros para o desenvolvimento da competência em matemática, proposto por M. Polanyi (Polanyi, 1962; Polanyi Society: www.muisc.edu/orgs/polanyi). 
Nunes, T. (1994). O papel da representação na resolução de problemas. Dynamis, 1, $19-27$.

Nunes, T. (1997). Systems Of signs and mathematical reasoning. Em T. Nunes \& P. Bryant (Org.), Learning and teaching mathematics: An international perspective. London: Psychology Press.

Nunes, T. (2000). How mathematics teaching develops pupils' reasoning systems. $9^{\text {th }}$ International Congress on Mathematics Education - [Abstracts]. Plenary Lectures And Regular Lectures, Tokio, Japan, 14-19.

Piaget, J. (1973a). A linguagem e as operações intelectuais. Em J. Ajuriaguerra, F. Bresson, P. Fraisse, B. Inheleder \& P. Oléron (Orgs.), Problemas de psicolingüística. São Paulo: Mestre Jou.

Piaget, J. (1973b). Biologia e conhecimento: Ensaio sobre as relações entre as regulacõoes orgânicas e os processos cognoscitivos. Petrópolis, RJ: Vozes.

Piaget, J. (1977). Psicologia da inteligência. Rio de Janeiro: Zahar.

Polanyi, M. (1962). Personal knowledge. London: Routledge \& Kegan Paul.

Schliemann, A. D., Santiago, M. M. L. \& Brito Lima, A. P. (1992). Understanding equivalences through balance scales. Proceedings of the XVI International Conference for the psychology of mathematics education. Durhan, New Hampshire.
Vergnaud, G., Cortes, A. E. \& Favre-Artigue, P. (1987). Introduction de l'algèbre auprès de débutants faibles: Problèmes épistémologiques et didactiques. Em G. Vergnaud, G. Brousseau \& M. Hulin (Orgs.), Didactique et acquisition des connaissances scientifiques: Actes du colloque de sèrres (pp. 259-280). Sèvres: La Pensée Sauvage.

Vergnaud, G. (1988). Theorical framewooks and empirical facts in the psychology of mathematics education. Proceedings of the International Congress on Mathematical Education, Budapest, pp. 39-41.

Vergnaud, G. (1990). La théorie des champs conceptuels. Recherches en Didactique des Mathématiques, 10, 133-170.

Vergnaud, G. (2000). Que peut apporter l'analyse de l'activité à la formation des enseignants et des formateurs? Carrefours de L'éducation, 10, 49-63.

Vigotski, L. S. (2001). A construção do pensamento e da linguagem. São Paulo: Martins Fontes.

Recebido: $17 / 11 / 2003$

$1^{a}$ revisão: $18 / 05 / 2004$

Aceite final: 29/07/2004

Sobre os autores

Mônica Maria Lins Lessa é Professora do Departamento de Educação da Universidade Federal Rural de Pernambuco, Pesquisadora do Laboratório de Pesquisa em Psicologia da Educação Matemática (LAPPEM), Recife (PE). É Doutora em Psicologia Cognitiva pela Universidade Federal de Pernambuco

Jorge Tarcísio da Rocha Falcão é Professor Adjunto do Departamento de Psicologia da Universidade Federal de Pernambuco, bolsista de Produtividade em Pesquisa do CNPq, vinculado ao Laboratório de Pesquisa em Psicologia da Educação Matemática (LAPPEM), Recife (PE). Doutor em Psicologia da Aprendizagem - Universidade de Paris-5. 